\title{
Nipple Sign, A Stigmata of Recent Arteriovenous Malformation Bleed in the Bronchial Tree
}

\author{
Rami Batarseh, MD'1, Yousef Shweihat, MD
}

ABSTRACT

Hemoptysis is defined as the expectoration of blood originating from the lower respiratory tract (i.e., below the vocal cords). Among the potential sources of bleeding from the bronchial tree are arteriovenous malformations (AVMs). Although pulmonary AVM and its diagnostic imaging features have been well described, less is known about bronchial AVM. This report describes an elderly man with multiple AVMs in his gastrointestinal tract who was admitted with hemoptysis and found to have AVM in his bronchial tree.
Author affiliations are listed at the end of this article.

Correspondence to: Rami Batarseh, MD Marshall Unviersity Joan C. Edwards School of Medicine batarseh@marshall.edu

\section{KEYWORDS}

nipple sign, hemoptysis, AVM

\section{INTRODUCTION}

Nipple sign is an indicator of recent bleeding in the gastrointestinal tract and is defined as an elevation in the mucosa with two types described: red nipple with a platelet plug and white nipple with a fibrin clot. ${ }^{1,2}$ Although arteriovenous malformations (AVMs) are also potential sources of bleeding from the bronchial tree, ${ }^{3}$ to our knowledge, nipple sign has never been reported in the bronchial tree. This report describes a patient with a nipple sign in the bronchial tree, indicating recent bleeding from an AVM.

\section{CASE PRESENTATION}

A 64-year-old man with a medical history of recurrent gastrointestinal bleeding presented to the emergency department after coughing up fresh blood that morning. He denied any prior history of similar symptoms. He reported feeling full in the chest, followed by coughing up fresh blood with no clots. The patient was a lifelong nonsmoker, with no occupational exposure to hazardous material, and no constitutional symptoms. A review of all systems was unremarkable.

Physical examination upon presentation yielded results typical for his age, with his vital signs being within normal limits. Laboratory workup, including coagulation profile, was unremarkable. Findings on chest $x$-rays and chest computed tomography (CT) were also unremarkable. Bronchoscopy was performed, showing abnormal findings in segment R7B (Figure 1). The patient was diagnosed with pulmonary AVM with a nipple sign consistent with recent bleeding. He was managed by direct cauterization. Follow-up at the clinic one and three months after discharge showed no recurrence of symptoms.

\section{DISCUSSION}

Pulmonary AVMs (PAVMs) are abnormal communications between pulmonary arteries and veins. Although uncommon, PAVMs should be considered in the differential diagnosis of common 


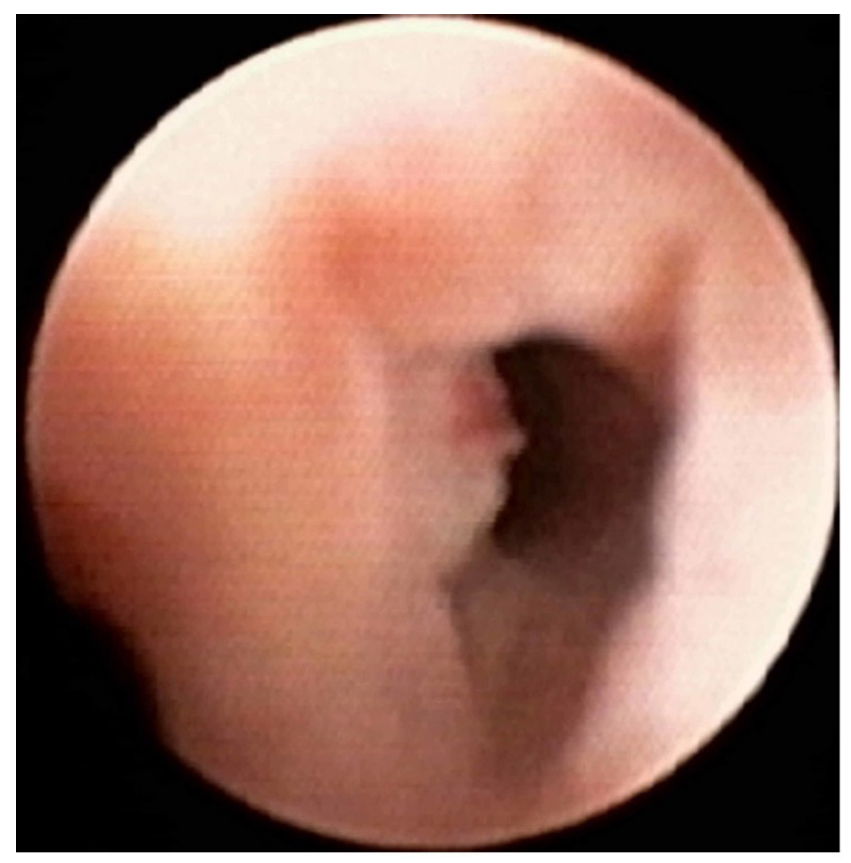

FIGURE 1: Bronchoscopic detection of a nipple sign in segment R7B.

pulmonary problems, including hypoxemia, pulmonary nodules, and hemoptysis. ${ }^{4}$

Dyspnea is the most common symptom of PAVMs, reported in as many as $50 \%$ of patients..$^{5}$ Other symptoms include hemoptysis in $7 \%$ to $30 \%$ of patients, as well as platypnea and orthodoxia, chest pain, cough, cyanosis, and murmurs. PAVMs are associated with other diseases, including hereditary hemorrhagic telangiectasia, which we believe affected our patient, as well as hemothorax, polycythemia, anemia, and pulmonary hypertension. ${ }^{6}$ Nodules on chest $\mathrm{x}$-rays or CT scans are the most common findings. CT angiography remains the gold standard for diagnosis, with magnetic resonance angiography having less diagnostic value. Bronchoscopy, which can directly visualize AVMs in the bronchial tree, is a valid diagnostic and therapeutic option. Although nipple sign has been reported in patients with gastrointestinal AVM bleeding, it has not, to our knowledge, been described in patients with AVM of the bronchial tree.

The presentation and workup of the present patient were unremarkable. He reported a history of recurrent gastrointestinal bleeding secondary to AVM. Bronchoscopy using an ultrathin bronchoscope for a detailed screening of smaller airways was both diagnostic and therapeutic, with cauterization being performed successfully.

Nipple sign in the Gl tract is a sign of recent bleeding, and disturbing this site is associated with further, potentially life-threatening bleeding. ${ }^{2}$ As it is not described in the bronchial tree, bronchoscopists should be aware of nipple sign and be able to identify it. Further information that includes various interventions is warranted to help reduce risks of acute and chronic bleeding.

\section{CONCLUSION}

AVM is an uncommon cause of hemoptysis but can be life-threatening. If imaging modalities fail to identify the potential source of bleeding, bronchoscopy is of great benefit both diagnostically and therapeutically. Bronchial AVM should be included in the differential diagnosis of patients with a similar presentation.

\section{AUTHOR AFFILIATIONS}

1. Marshall University Joan C. Edwards School of Medicine, Huntington, West Virginia

\section{REFERENCES}

1. Khan NM, Shapiro AB. The white nipple sign: please do not disturb. Case Rep Gastroenterol. 2011;5:386-390.

2. Abby Philips C, Sahney A. Oesophageal and gastric varices: historical aspects, classification and grading: everything in one place. Gastroenterol Rep. 2016;4:186-195.

3. Ingbar DH, Weinberger SE. Etiology of hemoptysis. [Internet] 2020. [Cited April 11, 2020] Available from: https://www.uptodate. com/contents/etiology-of-hemoptysis

4. Gossage JR: Pulmonary arteriovenous malformations: clinical features and diagnostic evaluation in adults. [Internet] 2020 [Cited April 11, 2020] Available from: https://www.uptodate. com/contents/pulmonary-arteriovenousmalformations-clinical-features-and-diagnostic- 
evaluation-in-adults

5. Cottin V, Chinet T, Lavolé A, et al.: Pulmonary arteriovenous malformations in hereditary hemorrhagic telangiectasia: a series of 126 patients. Medicine. 2007;86:1-17.

6. Shovlin CL, Tighe HC, Davies RJ, Gibbs JS, Jackson JE: Embolisation of pulmonary arteriovenous malformations: no consistent effect on pulmonary artery pressure. Eur Respir J. 2008;32:162-169. 